\title{
Structural mechanism of Bax inhibition by cytomegalovirus protein vMIA
}

\author{
Junhe Ma ${ }^{a}$, Frank Edlich ${ }^{b}$, Guillermo A. Bermejo ${ }^{a}$, Kristi L. Norris ${ }^{b}$, Richard J. Youle ${ }^{b}$, and Nico Tjandra ${ }^{a, 1}$ \\ a'Laboratory of Molecular Biophysics, National Heart, Lung, and Blood Institute, and 'burgical Neurology Branch, National Institute of Neurological Disorders \\ and Stroke, National Institutes of Health, Bethesda, MD 20892
}

Edited by Philippa Marrack, Howard Hughes Medical Institute, National Jewish Health, Denver, CO, and approved November 7, 2012 (received for review October 2, 2012)

The human protein Bax sits at a critical regulatory junction of apoptosis, or programmed cell death. Bax exists in equilibrium between cytosolic and mitochondria-associated forms that shifts toward the latter when Bax is activated by proapoptotic proteins. Activated Bax changes conformation, inserts into the mitochondrial outer membrane (MOM), oligomerizes, and induces MOM permeabilization, causing the release of cytochrome $c$, which effectively commits the cell to die. Because apoptosis is also a basic defense mechanism against invading pathogens, many viruses have developed counteractive measures. Such is the case of human cytomegalovirus, the replication of which hinges on VMIA (viral mitochondria-localized inhibitor of apoptosis), a virus-encoded protein with a unique, albeit poorly understood antiapoptotic activity by which it binds and recruits Bax to mitochondria. Here we show, via the structure determination of the complex between Bax and a peptide comprising VMIA's Bax-binding domain, that vMIA contacts Bax at a previously unknown regulatory site. Notably, using full-length vMIA, the structure is independently confirmed by assays in human cells that measure Bax subcellular localization and cytochrome $c$ release. Mutants that disrupt key intermolecular interactions disfavor VMIA's mitochondrial recruitment of Bax, and increase cytochrome $c$ release upon apoptosis induction. In a more stringent test, an engineered binding interface that achieves wild-type-like charge complementarity, although in a reversed fashion, recovers wild-type behavior. The structure suggests that by stabilizing key elements in Bax needed to unravel for its MOM insertion and oligomerization, vMIA prevents these important steps in apoptosis.

A poptosis, or programmed cell death, is a process essential for organ development and tissue maintenance (1-3) that, when dysfunctional, can contribute to several diseases, including cancer and autoimmunity (4). The protein Bax plays a crucial role in apoptosis in that its activation defines the fate of the cell (5): Bax translocates from the cytosol to mitochondria, accompanied by dramatic conformational changes, oligomerization, and the permeabilization of the mitochondrial outer membrane (MOM), which, in turn causes the release of cytochrome $c$ that activates the cell-demolition machinery. Bax's function is modulated by both pro- and antiapoptotic proteins from the Bcl-2 family (6). Members of this family share at least one Bcl-2 homology (BH) domain, and can be classified into three different groups: $(i)$ antiapoptotic proteins with four $\mathrm{BH}$ units (BH1234) (e.g., Bcl-2 and $\mathrm{Bcl}-\mathrm{x}_{\mathrm{L}}$ ), (ii) proapoptotic proteins with three $\mathrm{BH}$ units (BH123) (e.g., Bax and Bak), and (iii) proapoptotic BH3only proteins (e.g., Bid and Bim). The latter transmit prodeath signals, which, unless neutralized by the antiapoptotic Bcl-2 members, activate Bax (or Bak).

Many viruses have evolved mechanisms to inhibit apoptosis, used by the host organism to defend itself against infection (7). Indeed, the replication of human cytomegalovirus - a widely spread herpesvirus, pathogenic in immunocompromised individuals-relies on the encoded antiapoptotic protein vMIA (viral mitochondrialocalized inhibitor of apoptosis) (8-10). In contrast with antiapoptotic Bcl-2 members, which inhibit Bax mitochondrial localization, vMIA recruits Bax to mitochondria as part of its apoptotic inhibitory activity (11). Although previous structural determination efforts have provided invaluable atomistic insights about the interactions of $\mathrm{Bcl}-2$ proteins (see below), little is known with regards to that between Bax and vMIA.

In solution, Bax is $\alpha$-helical (Fig. 1) and displays its C-terminal helix, $\alpha 9$, lodged within a hydrophobic pocket (12). Experimental truncation of this helix in homologous (but functionally opposite) $\mathrm{Bcl}-\mathrm{x}_{\mathrm{L}}$ exposes the pocket, which binds peptides from $\mathrm{BH} 3$ domains of proapoptotic Bcl-2 members Bak, Bad, and Bim (5). Despite the existence of this canonical $\mathrm{BH} 3$ domain-binding site, structural studies on the complex of full-length Bax and a Bim$\mathrm{BH} 3$ peptide have revealed a different binding target, involving Bax helices $\alpha 1$ and $\alpha 6$, antipodal to the hydrophobic pocket, still occupied by $\alpha 9$ (13) (Fig. 1). Helix $\alpha 9$ is believed to be part of a C-terminal membrane-anchoring region, and is crucial for mitochondrial localization of Bax in normal cells. In cytomegalovirus-infected cells Bax is also recruited to mitochondria by vMIA. This process, however, is independent of $\alpha 9$ (14), which suggests the canonical BH3-binding site excludes the vMIA-Bax interaction surface. Furthermore, assuming the aforementioned Bax Bim-BH3 binding site (13) is shared with other BH3-only proteins, such as tBid (truncated Bid), this site also excludes vMIA, as tBid and vMIA do not compete for Bax as a binding partner (11).

Taken together, the above evidence suggests vMIA, with its unique antiapoptotic activity, binds Bax at a unique site. Indeed, this finding is confirmed here at atomic detail via the solution NMR structure determination of the complex between Bax and vMIA's Bax-binding domain (vMIA-BBD). The structure is additionally supported by mutations of residues identified to be important for the stability of the complex. The effect of the mutations is assessed in solution on the binding affinity between Bax and vMIA-BBD, as well as in human cells (using full-length vMIA) by the quantification of both the subcellular localization of Bax and the release of cytochrome $c$ upon apoptosis induction. The structure of the complex not only suggests the mechanism by which vMIA inhibits the activation of Bax, but also helps dissect even further the different interactions that converge on this crucial player in apoptosis.

\section{Results}

NMR Structure Determination of the Bax-vMIA-BBD Complex. The vMIA-BBD construct in our structural studies comprises residues

Author contributions: R.J.Y. and N.T. designed research; J.M., F.E., G.A.B., and K.L.N. performed research; J.M., G.A.B., and N.T. analyzed data; and J.M., G.A.B., and N.T. wrote the paper

The authors declare no conflict of interest.

This article is a PNAS Direct Submission.

Data deposition: The atomic coordinates and NMR restraints have been deposited in the Protein Data Bank, www.pdb.org (PDB ID code 2LR1).

${ }^{1}$ To whom correspondence should be addressed. E-mail: tjandran@nhlbi.nih.gov.

This article contains supporting information online at www.pnas.org/lookup/suppl/doi:10. 1073/pnas.1217094110/-/DCSupplemental. 

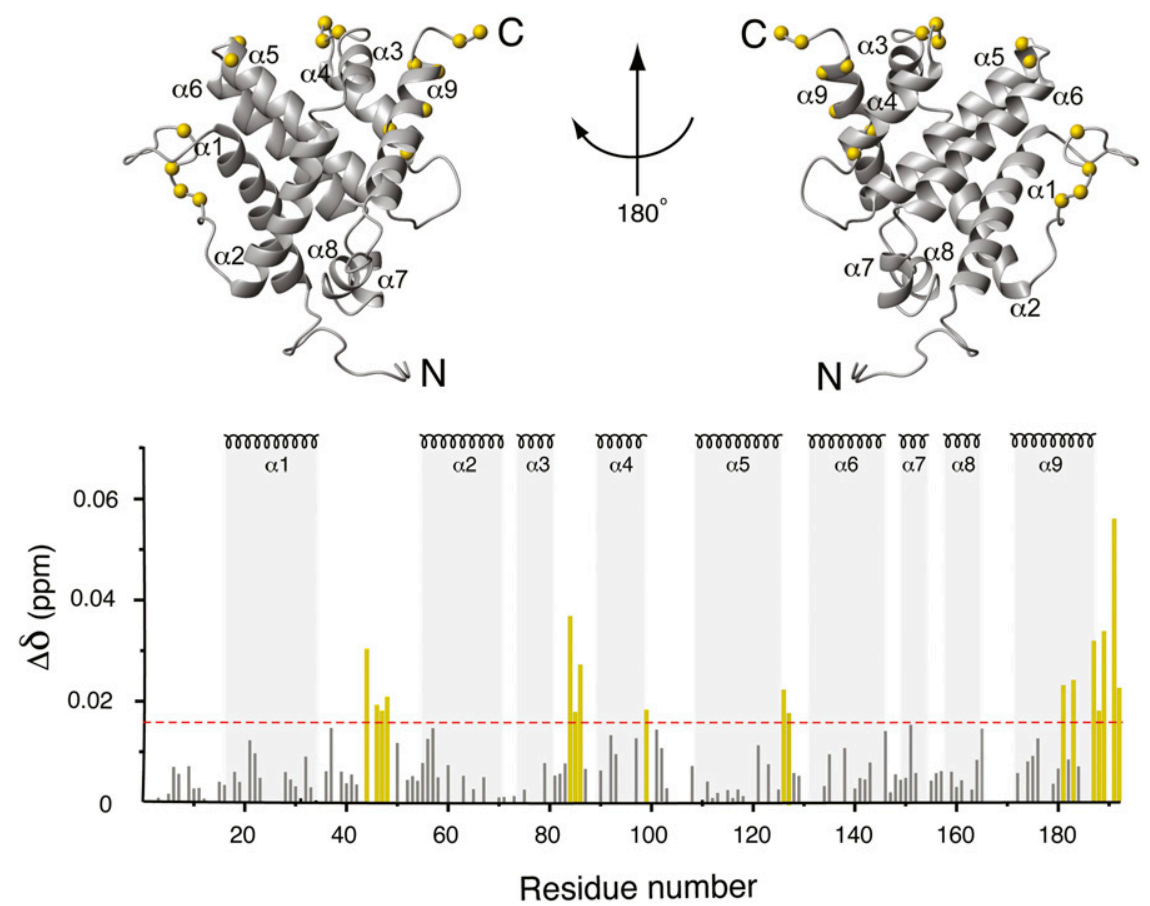

Fig. 1. Chemical-shift mapping of the vMIA-BBD binding site on Bax. The chemical-shift change of ${ }^{1} \mathrm{H}$ and ${ }^{15} \mathrm{~N}$ signals of ${ }^{15} \mathrm{~N}$-enriched Bax upon addition of vMIA-BBD, $\Delta \delta$ (Materials and Methods), is displayed against the structure of Bax (Upper) and versus residue number (Lower). $\alpha$-Helical segments are indicated throughout. Prolines and residues with overlapped NMR signals are not represented in the bar plot. Residues with chemical shift changes larger than 0.017 ppm (horizontal dashed line) are denoted by yellow bars, the corresponding $\mathrm{C}^{\alpha}$ atoms indicated by yellow spheres in the structure.

131-150 (with one exception; see below), the shortest sequence that displays full affinity to Bax (11). NMR structural characterization of this 20-aa peptide relied on nuclear Overhauser effects (NOEs), and reveals an $\alpha$-helical conformation from R139 to Q145, with backbone precision of $0.14 \AA$ for this region (Fig. S1). Such vMIA-BBD structure, along with that previously reported on Bax (12) (Fig. 1), provides the starting point for the determination of their complex.

The vMIA-binding surface on Bax was readily mapped by the perturbation of signals from the heteronuclear single-quantum coherence (HSQC) spectrum of Bax upon addition of vMIA$\mathrm{BBD}$, revealing four regions significantly affected (Fig. 1). Three segments, E44-D48, D84-D86, and C126-T127, correspond to the flexible loops between helices $\alpha 1-\alpha 2, \alpha 3-\alpha 4$, and $\alpha 5-\alpha 6$, respectively. A fourth region (residues L181, A183, and I187G192) contains part of helix $\alpha 9$ and the $\mathrm{C}$ terminus. Although distant in the amino acid sequence, these regions are close in space (Fig. 1) and cover a surface different from that of the canonical $\mathrm{BH} 3$-binding pocket (5) and the Bim-BH3 binding site (13) (Fig. S2). It is noteworthy that the overall modest signal perturbations do not imply weak binding (binding affinities are discussed in the following section), but likely the effect of competing processes, such as aggregation, which is observed rapidly $(2-3 \mathrm{~h})$ after addition of vMIA-BBD to Bax. Precedent for this phenomenon has been set by studies on the complex of Bax with a Bim-BH3 peptide (13).

The formation of aggregates degrades NMR spectral quality, allowing only short experiments such as the HSQC to be conducted, as opposed to the time-consuming ones at the heart of traditional structural NMR. Consequently, elucidation of the exact position of VMIA-BBD relative to Bax relied on HSQCbased paramagnetic relaxation enhancements (PREs) (15). HSQC spectra of Bax (vMIA-BBD) were acquired in presence of vMIA-BBD (Bax) derivatized with methanethiosulphonate (MTSL), whose paramagnetic nitroxide group broadens signals in a distance-dependent manner. The signal intensity of a nucleus close to the paramagnetic, oxidized nitroxide group $\left(\mathrm{I}_{\text {para }}\right)$ decreases relative to that in the diamagnetic, reduced state $\left(I_{\text {dia }}\right)$, and the $\mathrm{I}_{\text {para }} / \mathrm{I}_{\text {dia }}$ ratio provides intermolecular distance information (16).

MTSL was attached at four different positions (one at a time) along the length of vMIA-BBD via either native or engineered cysteines. The former, $\mathrm{C} 130$, is one residue off the $\mathrm{N}$-terminal end of the 131-150 peptide used in all other structural studies described herein. The engineered cysteines are A136C, F142C, and $\mathrm{Q} 145 \mathrm{C}$. The $\mathrm{I}_{\text {para }} / \mathrm{I}_{\text {dia }}$ profile of Bax corresponding to tag position $\mathrm{C} 130$ is shown in Fig. $2 A$, and shows slight differences relative to those produced by the other tagging sites in vMIABBD (Fig. S3), possibly because of averaging caused by the high flexibility the tag experiences at the terminal ends of the peptide. On the reverse paramagnetic tagging scheme, Bax C62S mutant was derivatized with MTSL at its remaining native C126, located in helix $\alpha 5$, and fortuitously proximal to the mapped binding surface. The resulting $\mathrm{I}_{\text {para }} / \mathrm{I}_{\text {dia }}$ profile of vMIA-BBD reflects a clear polarity (Fig. $2 B$ ), suggesting the $\mathrm{N}$ terminus of the peptide is closer to the paramagnetic tag.

Each PRE-dependent $\mathrm{I}_{\text {para }} / \mathrm{I}_{\text {dia }}$ intensity ratio was translated into a distance restraint between the corresponding proton and the MTSL tag (16), and used along with ambiguous distance restraints derived from the chemical shift perturbation data (17) (Fig. 1) to calculate the Bax-vMIA-BBD complex. The resulting ensemble of structures (Fig. S4) shows good convergence, with a precision around mean coordinates of $1.69 \pm 0.85 \AA$ for backbone and $1.79 \pm 0.63 \AA$ for all heavy atoms (considering only residues 16-35, 54-99, 108-188 of Bax, and 139-145 of vMIABBD). The structure ensemble has been deposited in Protein Data Bank under accession number 2LR1.

Effect of Mutations on the Binding Affinity of Bax for VMIA-BBD. vMIA-BBD binds opposite to the $\mathrm{BH} 1$ and $\mathrm{BH} 2$ domains of Bax, with its $\mathrm{N}$ terminus close to the $\mathrm{BH} 3$ domain (Fig. $3 A$ ), a previously unknown binding site, as expected from the chemical-shift data (Fig. 1). The peptide resides in a pocket outlined 


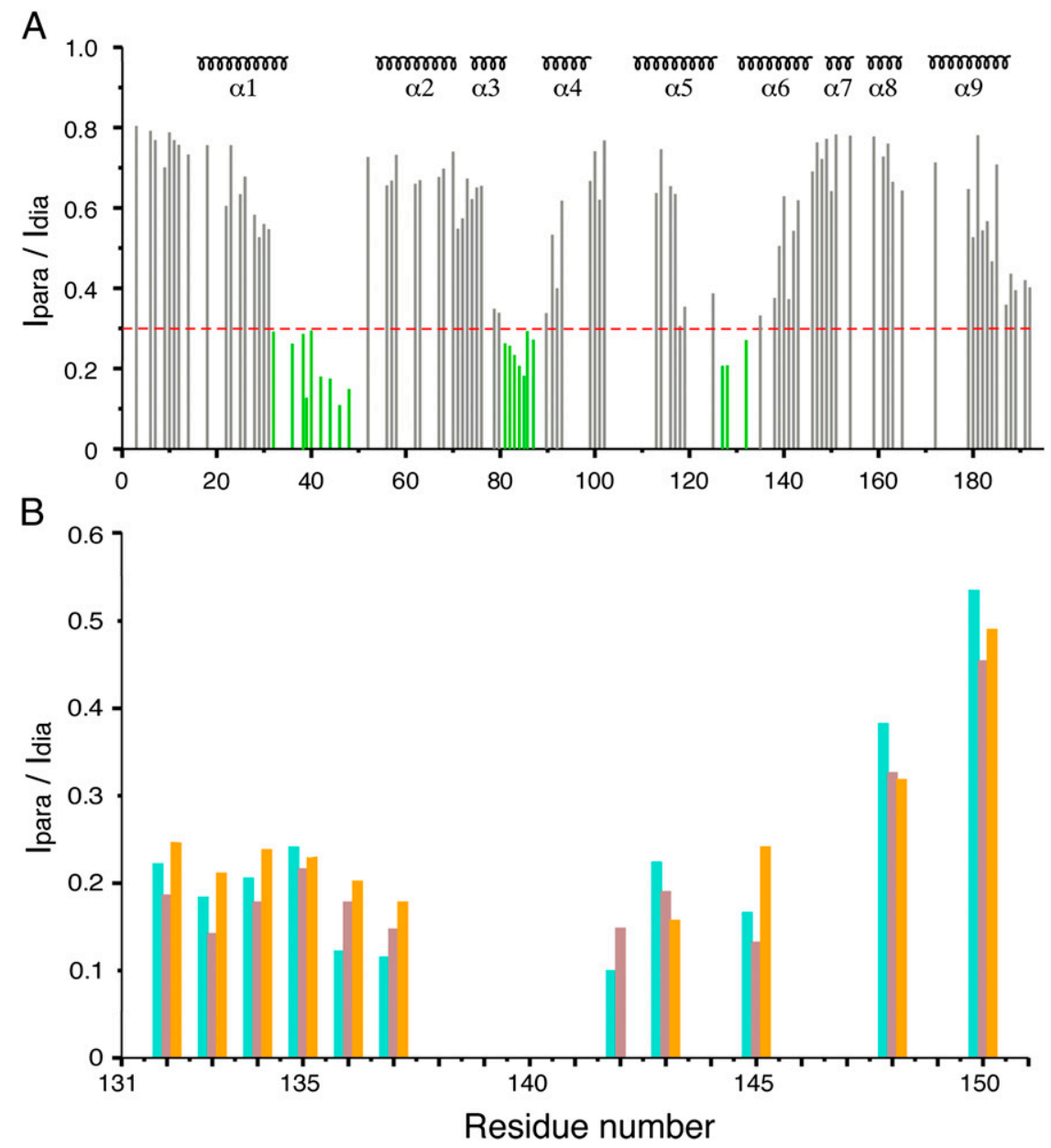

Fig. 2. Intermolecular paramagnetic relaxation enhancement. $(A)$ Residue profile for the ratio of backbone $H^{\mathrm{N}}$ signal intensity $\left(I_{\text {para }} / I_{\text {dia }}\right)$ of Bax's ${ }^{15} \mathrm{~N}$-HSQC spectrum in presence of paramagnetic/diamagnetic VMIA-BBD tagged with MTSL at C130. The secondary structure of Bax is indicated on top. (B) Same as in $A$ except that the $\mathrm{I}_{\text {para }} / \mathrm{I}_{\text {dia }}$ ratios stem from $\mathrm{H}^{\mathrm{N}}, \mathrm{H}^{\alpha}$, and $\mathrm{H}^{\beta}$ signals (cyan, brown, and orange bars, respectively) from ${ }^{15} \mathrm{~N}-$ and ${ }^{13} \mathrm{C}-\mathrm{HSQC}$ spectra of $\mathrm{vMIA-BBD}$ selectively ${ }^{15} \mathrm{~N} /{ }^{13} \mathrm{C}$-enriched (Ala, Leu, Lys, Phe, and Gln residues only), in the presence of Bax C62S MTSL-tagged at C126.

by Bax helices $\alpha 3-\alpha 4$ and $\alpha 5-\alpha 6$ (Fig. 3B), with its L143 facing a hydrophobic patch formed by Bax's V129, P130, L132, and I133 (Fig. 3C). Fluorescence anisotropy indicates mutation L143A decreases the binding affinity more than 20 -fold relative to the wild-type vMIA peptide (Table 1), which highlights the importance of this specific hydrophobic contact. Furthermore, favorable electrostatic interactions contribute to the stabilization of the complex, such as those between Bax's D84 and D86, and oppositely charged vMIA's R139 and R146 (Fig. 3C). Notably, changing the polarity of vMIA's R146 by mutation R146E, results in a 16-fold decrease in affinity for wild-type Bax; the affinity restored, albeit partially, by Bax D86K, which complements vMIA's R146E charge substitution (Table 1). Additionally reversing the charge of Bax's D84 (to yield Bax D84K/D86K) produces a small further affinity increase, consistent with its more distal location to vMIA's R146. Fluorescence anisotropy curves are shown in Figs. S5 and S6.

Effect of Mutations on the Activity of Full-Length vMIA in Human Cells. The importance of the above-discussed electrostatic interactions was confirmed in human cells with full-length vMIA, using HCT116 Bax/Bak double-knockout cells overexpressing vMIA and C-terminal-truncated $\operatorname{Bax}(\operatorname{Bax} \Delta C)(18)$. As shown in Fig. $4 A$ and $B$, wild-type $\operatorname{Bax} \Delta C$ and double-mutants D84S/ D86S, and D84K/D86K are predominantly cytosolic in absence of vMIA. Upon wild-type vMIA coexpression, wild-type Bax $\Delta \mathrm{C}$ appears mostly mitochondria-associated, but the two variants lacking the wild-type aspartic residues show mixed cytosolic/mitochondrial localization indicative of a deficient Bax-vMIA affinity. Cells expressing wild-type $\operatorname{Bax} \Delta \mathrm{C}$ with vMIA R139E/ $\mathrm{R} 146 \mathrm{E}$ share such mixed pattern. The combination of $\mathrm{Bax} \Delta \mathrm{C}$ D84S/D86S and vMIA R139E/R146E significantly abolishes vMIA's recruitment of Bax to mitochondria, consistent with a significantly disrupted affinity. More importantly, however, the recruiting activity of vMIA R139E/R146E is restored in the presence of Bax $\Delta \mathrm{C}$ D84K/D86K, where the charge complementarity across the binding interface observed in the complex (Fig. $3 C$ ) has been reinstated, albeit in a reversed fashion. The observed subcellular distribution patterns of $\operatorname{Bax} \Delta C$ are consistent with assays that monitor cytochrome $c$ release after apoptosis induction using full-length Bax (Fig. 4C). The charge complementary mutants (Bax D84K/D86K and vMIA R139E/R146E) show low, wild-type levels of cytochrome $c$, indicating the full protective capacity of the complex.

\section{Discussion}

Isolated mitochondria assays have previously shown (11) that Bax oligomers, a hallmark of apoptosis, overcome vMIA's function. Similarly, Bax activated by tBid [which is known to cause Bax oligomerization (19)] also overcomes the protective effect of vMIA (11). These findings suggest that the vMIA-binding site is not available in oligomeric Bax. The most detailed study on Bax 

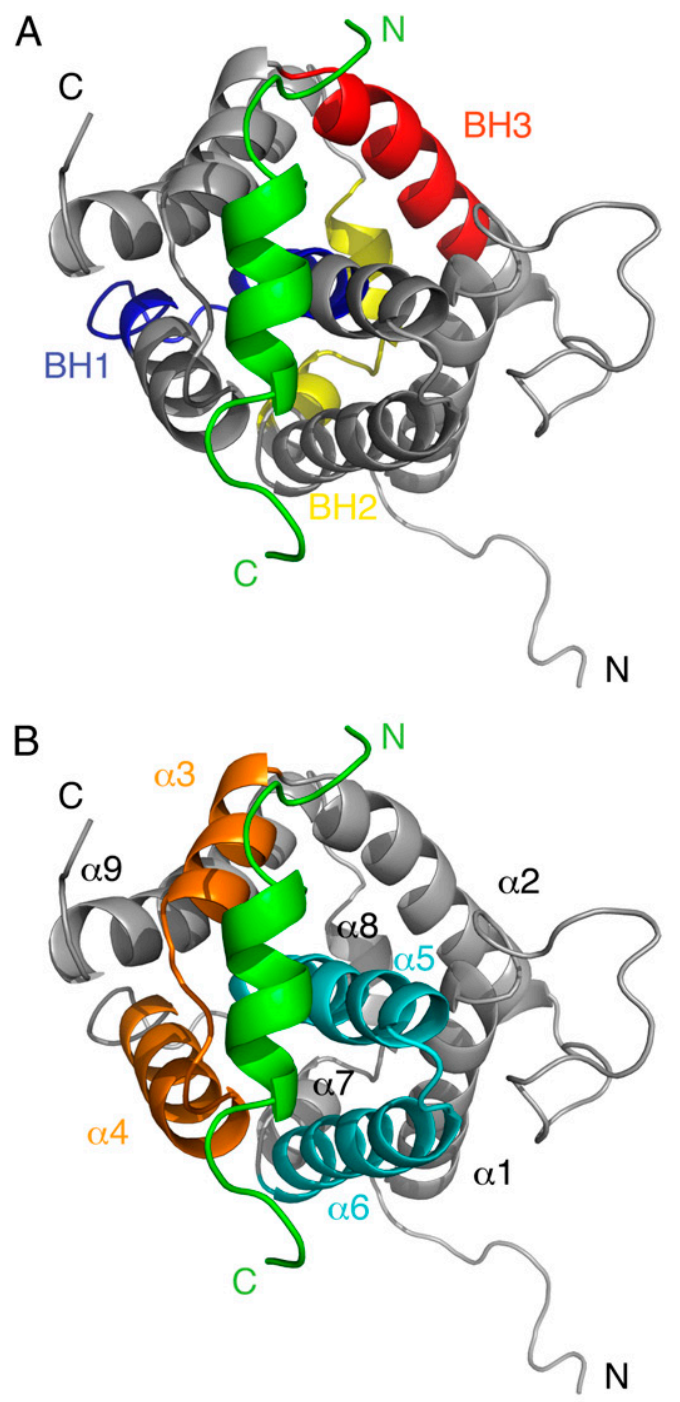

C

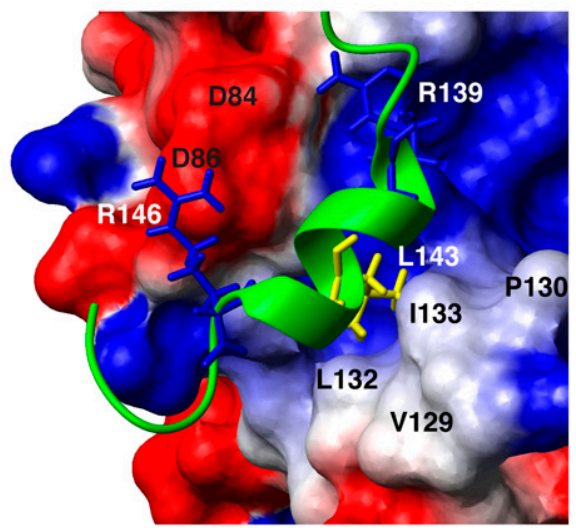

Fig. 3. Bax-VMIA-BBD complex. $(A$ and $B$ ) Ribbon display of a representative structure of the complex, with VMIA-BBD colored green. The $\mathrm{N}$ and $\mathrm{C}$ termini are indicated. $(A) \mathrm{BH}$ domains of Bax are indicated. $(B)$ Helices of Bax are indicated, with the $\alpha 3 / \alpha 4$ and $\alpha 5 / \alpha 6$ hairpins highlighted in orange and cyan, respectively. (C) Close-up view of the binding pocket, where Bax is shown in a surface representation with positive (blue) and negative (red) charges indicated, as well as the position of selected residues (black labels). VMIA-BBD is shown as a green ribbon, with selected side chains (white labels) displayed in stick representation.
Table 1. Binding affinities of various forms of vMIA-BBD and Bax

\begin{tabular}{lcc} 
Bax & VMIA-BBD & $K_{d}(\mathrm{nM})^{*}$ \\
\hline Wild-type & Wild-type $\left({ }^{130}\right.$ CEALKKALRRHRFLWQRRQRA $\left.{ }^{150}\right)$ & $22 \pm 2$ \\
Wild-type & L143A & $491 \pm 20$ \\
Wild-type & R139E & $78 \pm 5$ \\
Wild-type & R146E & $352 \pm 14$ \\
D86K & R146E & $136 \pm 9$ \\
D84K/D86K & R146E & $105 \pm 9$ \\
\hline
\end{tabular}

*SDs were determined from three independent measurements of fluorescence anisotropy.

oligomerization to date has recently identified key residues via cysteine linkage experiments (20). R94 and V95 in helix $\alpha 4$ were implicated within a dimer interface, and residues I136, W139, D142, and E146, all in helix $\alpha 6$, engaged in aggregates of dimers. Interestingly, in monomeric, nonactivated Bax (Fig. 1), I136 is buried within helices $\alpha 4$ and $\alpha 6$ (among others), which suggests $\alpha 4$ and $\alpha 6$ have to come apart for high-order aggregates to occur. Moreover, the $\alpha 5 / \alpha 6$ hairpin is thought to integrate into the MOM upon Bax activation (21), which further advocates for the separation of $\alpha 6$ and nontransmembrane $\alpha 4$ during apoptosis. In this light, the structure of the Bax-vMIA-BBD complex, solved here by solution-state NMR and supported by mutational assays both in solution and in human cells, suggests that by simultaneously stabilizing the $\alpha 3 / \alpha 4$ and $\alpha 5 / \alpha 6$ hairpins, vMIA strategically positions itself to prevent the conformational changes Bax needs to undergo for its MOM insertion and oligomerization, events that invariably lead to the death of the cell. These insights not only open new doors for drug development against cytomegalovirus, but also provide a different perspective on the function of Bax in uninfected cells.

\section{Materials and Methods}

vMIA-BBD Peptide Preparation. Wild-type and site-specific mutants of natural isotopic abundance vMIA-BBD peptides were purchased from Bio-Synthesis. vMIA-BBD peptide ${ }^{13} \mathrm{C}^{15} \mathrm{~N}$-enriched at Ala, Leu, Lys, Phe, and Gln positions was purchased from AnaSpec. Two types of peptides, all N-acetylated and C-amidated, were used: a 21-residue version (Ac- ${ }^{130}$ CEALKKALRRHRFLWQRRQRA ${ }^{150}$ $\mathrm{CONH}_{2}$ ), or a 20-residue one that lacks C130.

Paramagnetic studies relied on single-cysteine VMIA-BBD, either the 21residue peptide with its native $\mathrm{C} 130$, or 20 -residue mutants at positions 136 , 142 , and 145, respectively. The free sulfhydryls were reacted with fivefold molar excess of MTSL (Toronto Research Chemicals) in DMSO for $30 \mathrm{~min}$ at room temperature. DMSO was removed by lyophilization, the residual powder resuspended in methanol, and the pure MTSL-tagged VMIA-BBD precipitated with ether/hexanes to remove excess unreacted spin label. Reaction completion was confirmed by liquid chromatography-mass spectrometry (LC-MS).

Fluorescence anisotropy experiments relied on native C130 (21-residue peptide), incubated with 10-fold molar excess of BIODIPY FL $N$-(2-aminoethyl) maleimide (Invitrogen) reagent in $50 \%$ (vol/vol) DMSO/ $\mathrm{H}_{2} \mathrm{O}$ overnight at $4{ }^{\circ} \mathrm{C}$, and then purified by reverse-phase HPLC (C4 column; Vydac). The organic solvent was removed by lyophilization, the residual powder resuspended in water, and subsequently dialyzed against $20 \mathrm{mM}$ potassium phosphate buffer at $\mathrm{pH} 6.2$.

Bax Preparation. Recombinant Bax for NMR experiments and biochemical/ biophysical assays was generated as previously described (12). Mutants were produced via PCR-based site-directed mutagenesis, subsequently verified by DNA sequencing. Bax MTSL-tagged at its native C126 was generated by initially treating the C62S mutant with 10-fold molar excess of DTT for $2 \mathrm{~h}$, subsequently desalted on a PD-10 column using $20 \mathrm{mM}$ potassium phosphate buffer at $\mathrm{pH}$ 6.2. After the desalting step, a 20-fold molar excess of MTSL was immediately introduced, followed by overnight incubation at $4{ }^{\circ} \mathrm{C}$ Excess unreacted MTSL was removed by a PD-10 column. The success of the paramagnetic tagging reaction was confirmed via LC-MS.

NMR Spectroscopy and Structure Calculation of vMIA-BBD. The NMR samples used for the structure determination of VMIA-BBD contained $1 \mathrm{mM}$ of natural 

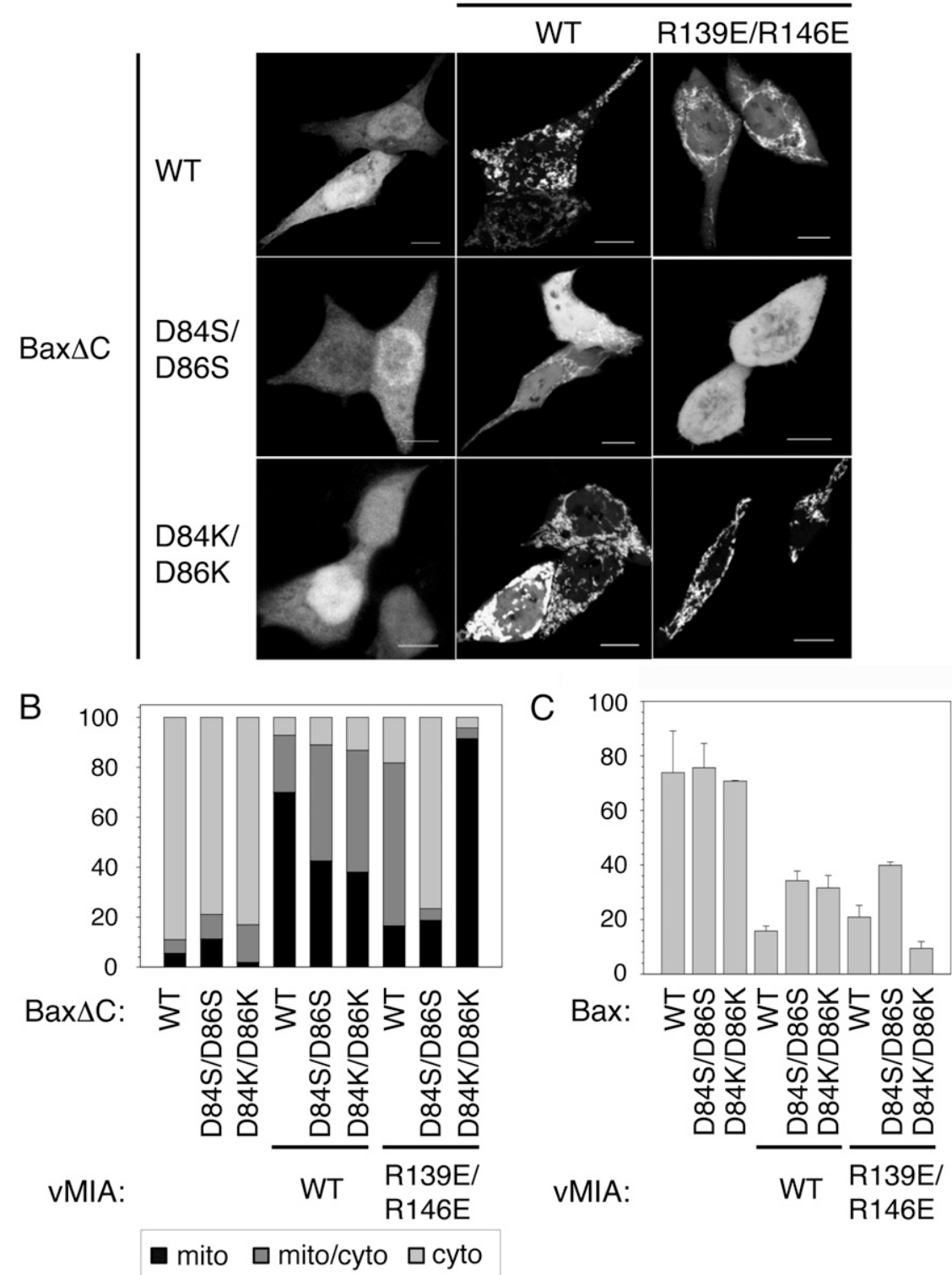

Fig. 4. Effect of VMIA on Bax subcellular localization and cytochrome $c$ release in HCT116 Bax/Bak double-knockout cells coexpressing Bax and vMIA variants. (A) Confocal microscopy indicates the localization of GFP-Bax $\Delta$ C. (Scale bars, $10 \mu \mathrm{m}$.) (B) Quantification of GFP-Bax $\Delta \mathrm{C}$ was done by evaluating 100 cells. Light gray, gray, and black bars correspond to the percentage of cell population where GFP-Bax $\Delta C$ was found in the cytosol, cytosol and mitochondria, and mitochondria only, respectively. (C) Percentage amount of cytochrome $c$ release after apoptosis induction with $1 \mu \mathrm{M}$ staurosporine in cells expressing full-length Bax.

isotopic abundance or selectively ${ }^{13} \mathrm{C} /{ }^{15} \mathrm{~N}$-enriched peptide in $20 \mathrm{mM}$ potassium phosphate buffer and $10 \%$ (vol/vol) $\mathrm{D}_{2} \mathrm{O}$ at $\mathrm{pH}$ 6.2. The spectra dataset for chemical-shift assignment of ${ }^{1} \mathrm{H},{ }^{15} \mathrm{~N}$, and ${ }^{13} \mathrm{C}$ signals, and struc tural characterization consisted of $\mathrm{CBCA}(\mathrm{CO}) \mathrm{NH}, \mathrm{HNCACB},{ }^{15} \mathrm{~N}$-separated NOESY, and homonuclear TOCSY, COSY, and NOESY, all experiments collected at $15^{\circ} \mathrm{C}$ on a Bruker $600 \mathrm{MHz}$ spectrometer.

The structure of the VMIA-BBD peptide was calculated by well-established distance geometry and simulated annealing protocols (22), using the program Xplor-NIH $(23,24)$, on the basis of 83 intra- and 73 interresidue proton-proton NOE distance restraints, as well as five hydrogen bond and five dihedral angle restraints within the helical region.

Fluorescence Anisotropy Assays. Fluorescence anisotropy was measured by adding Bax aliquots to a $25 \mathrm{nM}$ solution of VMIA-BBD in $20 \mathrm{mM}$ potassium phosphate buffer $(\mathrm{pH} 6.2)$ at $4{ }^{\circ} \mathrm{C}$. All measurements were performed using the PTI EasyLife LS fluorometer equipped with a Peltier cell holder and a doubledetection T-geometry system with Glan-Thompson polarizers. Fluorescence emission was detected at a right angle from the excitation beam. An excitation wavelength of $494 \mathrm{~nm}$ with bandwidth of $5 \mathrm{~nm}$ was used. Fluorescence emission of BODIPY was detected at a wavelength of $515 \mathrm{~nm}$ with a bandwidth of $5 \mathrm{~nm}$.

Chemical-Shift Mapping of the Bax-vMIA-BBD Binding Interface. All chemical shift mapping experiments were conducted at $32^{\circ} \mathrm{C}$ on a Bruker $800 \mathrm{MHz}$ spectrometer. The binding site on Bax was mapped by adding VMIA-BBD from 3-mM stock to a $0.15-\mathrm{mM}$ solution of ${ }^{15} \mathrm{~N}$-enriched Bax, to a 1:1 molar ratio, all solutions in $20 \mathrm{mM}$ potassium phosphate buffer and 10\% (vol/vol) $\mathrm{D}_{2} \mathrm{O}$ at $\mathrm{pH}$ 6.2. The weighted average chemical-shift difference, $\Delta \delta$, was calculated as $\left\{0.5\left[\left(\Delta \delta^{\mathrm{H}}\right)^{2}+\left(\Delta \delta^{\mathrm{N}} / 5\right)^{2}\right]\right\}^{1 / 2}$ (in ppm), where $\Delta \delta^{\mathrm{X}}$ is the difference for nucleus $\mathrm{X}$, measured from ${ }^{15} \mathrm{~N}-\mathrm{HSQC}$ spectra (25). Ambiguous distance restraints used for the structure calculation of the complex between Bax and VMIA-BBD (see below) were derived following previous work (17), considering Bax residues with both $\Delta \delta>0.017 \mathrm{ppm}$ and solvent accessibility over $45 \%$, as reported by the program NACCESS (26) on the known Bax structure (12). Such so-called active residues included T14, K21, R37, E44, 
A46, L47, D48, V50, K57, D84, T85, D86, A97, S101, D102, C126, G138, E146, W151, A183, I187, K189, M191, and G192. On the other hand, active residues of VMIA-BBD were K135, A136, L137, L143, and Q145, determined as those that experienced the largest chemical-shift perturbations upon addition of Bax to an $80-\mu \mathrm{M}$ solution of the selectively ${ }^{15} \mathrm{~N} /{ }^{13} \mathrm{C}$-enriched vMIABBD peptide.

Paramagnetic Relaxation Enhancement. Samples for NMR PRE measurements on Bax contained $0.15 \mathrm{mM}$ uniformly ${ }^{15} \mathrm{~N}$-enriched protein, and those for measurements on vMIA-BBD contained $0.10 \mathrm{mM}$ selectively ${ }^{15} \mathrm{~N} /{ }^{13} \mathrm{C}$-enriched peptide, all solutions in $20 \mathrm{mM}$ potassium phosphate buffer and $10 \%$ (vol/ vol) $\mathrm{D}_{2} \mathrm{O}$ at $\mathrm{pH}$ 6.2. In each case, natural abundance MTSL-tagged binding partner (titrant) was added to the solution of the isotopically enriched counterpart (analyte) to achieve a 1:1 analyte:titrant ratio. ${ }^{15} \mathrm{~N}-\mathrm{HSQC}$ spectra were recorded at $32{ }^{\circ} \mathrm{C}$ on a Bruker $800 \mathrm{MHz}$ spectrometer under both paramagnetic and diamagnetic conditions, the latter achieved by adding ascorbic acid (10-fold molar excess relative to the MTSL-tagged titrant) to the paramagnetic sample before spectral acquisition. For PRE measurements on vMIA-BBD, additional ${ }^{13} \mathrm{C}-\mathrm{HSQC}$ spectra were recorded. PRE effects were obtained from the ratio of integrated peak intensity in the paramagnetic and diamagnetic spectra $\left(I_{\text {para }} / l_{\text {dia }}\right)$. Such ratios were calculated after normalizing the intensities (to account for small differences in the paramagnetic and diamagnetic spectra) using peaks arising from unaffected residues, far from the binding site. Overlapped peaks were excluded from this analysis.

For the structure calculation of the complex (see below), $I_{\text {para }} / I_{\text {dia }}$ ratios were translated into distance restraints between the $S^{\gamma}$ of the cysteine where the MTSL resided and the corresponding protons, which in Bax consisted of those ${ }^{15} \mathrm{~N}$-attached, and in vMIA-BBD included both ${ }^{15} \mathrm{~N}$ - and ${ }^{13} \mathrm{C}$ attached. Ratios were grouped into four classes $\left(I_{\text {para }} / I_{\text {dia }}<0.1,0.1<I_{\text {para }} / I_{\text {dia }}<\right.$

1. Kerr JF, Wyllie AH, Currie AR (1972) Apoptosis: A basic biological phenomenon with wide-ranging implications in tissue kinetics. Br J Cancer 26(4):239-257.

2. Raff MC (1992) Social controls on cell survival and cell death. Nature 356(6368): 397-400.

3. Vaux DL, Haecker G, Strasser A (1994) An evolutionary perspective on apoptosis. Cell 76(5):777-779.

4. Thompson CB (1995) Apoptosis in the pathogenesis and treatment of disease. Science 267(5203):1456-1462.

5. Youle RJ, Strasser A (2008) The BCL-2 protein family: Opposing activities that mediate cell death. Nat Rev Mol Cell Biol 9(1):47-59.

6. Edlich $\mathrm{F}$, et al. (2011) $\mathrm{Bcl}-\mathrm{x}(\mathrm{L})$ retrotranslocates Bax from the mitochondria into the cytosol. Cell 145(1):104-116.

7. Tschopp J, Thome M, Hofmann K, Meinl E (1998) The fight of viruses against apoptosis. Curr Opin Genet Dev 8(1):82-87.

8. Goldmacher VS (2002) VMIA, a viral inhibitor of apoptosis targeting mitochondria. Biochimie 84(2-3):177-185.

9. Goldmacher VS, et al. (1999) A cytomegalovirus-encoded mitochondria-localized inhibitor of apoptosis structurally unrelated to Bcl-2. Proc Natl Acad Sci USA 96(22): 12536-12541.

10. McCormick AL, Skaletskaya A, Barry PA, Mocarski ES, Goldmacher VS (2003) Differential function and expression of the viral inhibitor of caspase 8-induced apoptosis (vICA) and the viral mitochondria-localized inhibitor of apoptosis (vMIA) cell death suppressors conserved in primate and rodent cytomegaloviruses. Virology 316(2): 221-233.

11. Arnoult D, et al. (2004) Cytomegalovirus cell death suppressor vMIA blocks Bax- but not Bak-mediated apoptosis by binding and sequestering Bax at mitochondria. Proc Natl Acad Sci USA 101(21):7988-7993.

12. Suzuki M, Youle RJ, Tjandra N (2000) Structure of Bax: Coregulation of dimer formation and intracellular localization. Cell 103(4):645-654.

13. Gavathiotis $E$, et al. (2008) BAX activation is initiated at a novel interaction site. Nature 455(7216):1076-1081.
$0.2,0.2<\mathrm{I}_{\text {para }} / I_{\mathrm{dia}}<0.3$, and $\left.0.3<\mathrm{I}_{\mathrm{para}} / \mathrm{I}_{\mathrm{dia}}<0.5\right)$, each associated with appropriate distance restraints bounds (16)

Structure Calculation of the Bax-vMIA-BBD Complex. The previously solved NMR structure of Bax (12) and that of VMIA-BBD determined here were docked via a conjoined rigid body/torsion angle dynamics protocol (17), using the chemical shift perturbation and PRE-based restraints. During the course of the structure calculations, which were performed with the Xplor-NIH software package $(23,24)$, the polypeptide backbone of all helical segments of Bax, as well as that of VMIA-BBD, was treated as a rigid body, giving the remainder full torsional degrees of freedom. Five-hundred structures were calculated from random initial positions of Bax and VMIA-BBD, from which the 20 lowestenergy ones were chosen as the representative ensemble.

Human Cell Assays. HCT116 Bax/Bak double-knockout cells were obtained, cultured, and transfected as described previously (6). Human GFP-tagged $\operatorname{Bax} \Delta \mathrm{C}$ has been described elsewhere (18), as well as the confocal microscopy procedure (6). Cytochrome $c$ release was determined by immunostaining (18); all quantification assays relied on the examination of 100 cells.

ACKNOWLEDGMENTS. We thank Motoshi Suzuki for assisting in the generation of Bax mutants and providing comments on this manuscript; Yi He for assisting in the fermentations to produce recombinant Bax; Grzegorz Piszczek (Biophysics Core Facility, National Heart, Lung, and Blood Institute, National Institutes of Health) for advising on fluorescence anisotropy; and Duck-Yeon Lee (Protein Analysis Core Facility, National Heart, Lung, and Blood Institute, National Institutes of Health) for advising on mass spectrometry. F.E. was supported by the Leopoldina, National Academy of Sciences, Germany. This work was supported by the Intramural Research Program of the National Institutes of Health, National Institute of Neurological Disorders and Stroke (R.J.Y.) and the National Heart, Lung, and Blood Institute (N.T.).

14. Poncet D, et al. (2004) An anti-apoptotic viral protein that recruits Bax to mito chondria. J Biol Chem 279(21):22605-22614.

15. Iwahara J, Tang $C$, Marius Clore G (2007) Practical aspects of ${ }^{(1)} \mathrm{H}$ transverse paramagnetic relaxation enhancement measurements on macromolecules. J Magn Reson 184(2):185-195.

16. Battiste JL, Wagner G (2000) Utilization of site-directed spin labeling and high-resolution heteronuclear nuclear magnetic resonance for global fold determination of large proteins with limited nuclear overhauser effect data. Biochemistry 39(18): 5355-5365.

17. Clore GM, Schwieters CD (2003) Docking of protein-protein complexes on the basis of highly ambiguous intermolecular distance restraints derived from $1 \mathrm{H} / 15 \mathrm{~N}$ chemical shift mapping and backbone $15 \mathrm{~N}-1 \mathrm{H}$ residual dipolar couplings using conjoined rigid body/torsion angle dynamics. J Am Chem Soc 125(10):2902-2912.

18. Norris KL, Youle RJ (2008) Cytomegalovirus proteins vMIA and m38.5 link mitochondrial morphogenesis to Bcl-2 family proteins. J Virol 82(13):6232-6243.

19. Desagher S, et al. (1999) Bid-induced conformational change of Bax is responsible for mitochondrial cytochrome c release during apoptosis. J Cell Biol 144(5):891-901.

20. Dewson G, et al. (2012) Bax dimerizes via a symmetric BH3:groove interface during apoptosis. Cell Death Differ 19(4):661-670.

21. Annis MG, et al. (2005) Bax forms multispanning monomers that oligomerize to permeabilize membranes during apoptosis. EMBO J 24(12):2096-2103.

22. Kuszewski J, Nilges M, Brünger AT (1992) Sampling and efficiency of metric matrix distance geometry: A novel partial metrization algorithm. I Biomol NMR 2(1):33-56.

23. Schwieters CD, Kuszewski JJ, Clore GM (2006) Using Xplor-NIH for NMR molecular structure determination. Prog Nucl Magn Reson Spectrosc 48(1):47-62.

24. Schwieters CD, Kuszewski JJ, Tjandra N, Clore GM (2003) The Xplor-NIH NMR molecular structure determination package. J Magn Reson 160(1):65-73.

25. Grzesiek S, et al. (1996) The solution structure of HIV-1 Nef reveals an unexpected fold and permits delineation of the binding surface for the $\mathrm{SH} 3$ domain of Hck tyrosine protein kinase. Nat Struct Bio/ 3(4):340-345.

26. Hubbard SJ, Thornton JM (1993) NACCESS Computer Program (Department of Biochemistry and Molecular Biology, Univ College London, London). 